\title{
Errata: Growth of Clusters in a First-Order Phase Transition ${ }^{1}$
}

\author{
O. Penrose, ${ }^{2}$ Joel L. Lebowitz, ${ }^{3}$ J. Marro, ${ }^{4}$ M. H. Kalos, ${ }^{5}$ and A. Sur ${ }^{6}$
}

Received July 23, 1980

The attention of the reader is drawn to several misprints which may obscure the understanding of our paper:

Page 251, Eq. (13) should read

$$
D=\Delta x^{2} /(6 \Delta t)=1 / 6
$$

Page 251, line 25 should read: " $2 F(0)=1$. The estimated value for $\alpha$ is therefore"

Page 251, Eq. (14) should read

$$
\alpha=4 \pi(1 / 6)(3 / 4 \pi)^{1 / 3}=1.3
$$

Page 252, Eq. (19) should read

$$
A=2.415(1-\rho)^{3} \alpha w_{s} \approx 0.026
$$

Page 263, line 9 should read: "formula for $A$ brings up the value of $A$ from 0.026 [as obtained in Eq. (19)]"

Page 263, line 10 should read: "to a value in the range $0.04-0.08$. Unfortunately, the observed value of $A$ "

\footnotetext{
' This paper appeared in J. Stat. Phys. 19:243 (1978).

${ }^{2}$ Faculty of Mathematics, The Open University, Milton Keynes, England.

${ }^{3}$ Department of Mathematics, Rutgers University, New Brunswick, N.J.

${ }^{4}$ Departamento de Física Teórica, Facultad de Física de la Universidad de Barcelona, Becario de la Foundación March, Spain.

${ }^{5}$ Courant Institute of Mathematical Sciences, New York University, New York, N.Y.

${ }^{6}$ Boeing Computer Services, New York, N.Y.
} 